\title{
The interface theory of perception leaves me hungry for more: Commentary on Hoffman, Singh, and Prakash, "The interface theory of perception"
}

\author{
Matthew Schlesinger
}

Published online: 18 September 2015

(C) Psychonomic Society, Inc. 2015

\begin{abstract}
The interface theory offers a rich blend of logic and mathematical modeling with a dash of evolutionary storytelling, leading to the conclusion that perceptual experience and physical reality are only loosely related. Is the theory convincing? I would have to say "almost"; although it certainly has many elements working in its favor, ultimately, I also found that some important questions were ignored or left unanswered (e.g., a more fully articulated account of how evolutionary mechanisms operate on perception). I am quite optimistic that the next iteration of the theory will be able to address these issues.
\end{abstract}

Keywords Evolutionary mechanisms $\cdot$ Mathematical models · Phenomenalism

Hoffman, Singh, and Prakash (2014) have ventured into dangerous waters by articulating the idea that perceptual experience is not (necessarily) veridical. They provide support for their provocative claim in a variety of ways (some more convincing than others), but are still careful to remind the astonished reader that even though you may not perceive the objective reality of your neighbor's car, you ought not to steal it. Just because the "true" car cannot be objectively perceived does not mean that it isn't solid and real, and very much another person's property.

Overall, I found the argument to be a superb opening shot in what may grow to become a rather contentious debate. I admired and appreciated seeing (or should I say "seeing"?) many important elements of their argument. On the other hand, despite what appears to be a considerable amount of effort to establish the foundation for their theory, they have

M. Schlesinger $(\bowtie)$

Department of Psychology, Southern Illinois University Carbonale, Carbondale, IL 62901, USA

e-mail: matthews@siu.edu also left a number of rather important unanswered questions that I hope will be addressed at some point. I highlight here what I found utterly compelling, as well as not completely satisfying, about their account.

\section{We got phylogeny and ontogeny!}

What a delight to discover that the interface theory of perception not only includes the evolutionary timescale, but also makes room for the developmental timescale. In particularas I highlight below-although the notion of perceptionaction coupling is brought into the theory in order to create an explicitly active observer, an added benefit is that the same mechanism is presumed to work on both the phylogenetic and ontogenetic playing fields. This is an elegant yet relatively inexpensive assumption, though unfortunately it is not systematically explored in the analysis and makes only a brief appearance. Indeed, I wonder whether the authors are fully aware of the implications. At the very least, this feature means that there is a potential continuity across the two timescales, opening the door to a discussion of multitimescale interactions, including exotic subjects such as heterochrony and the Baldwin effect.

\section{Perception meets action}

Another appealing feature of the interface theory is the idea that perception is not idiosyncratic and completely subjective, but is linked to reality through perception-action couplings, loops, or mappings. In other words, because the laws of physics apply to the movements and actions of physical objects (including such items as eyes, ears, arms, and legs), walking around, kicking tires, and generally manipulating the environment has lawful consequences. Actions (within a class) produce predictable perceptual experiences (within a 
corresponding class) of perceptual effects. This claim feels very Gibsonian to me, though it is clear that Hoffman et al. would prefer to keep their distance from the ecological theory of perception. Perhaps it is safe to say that both theories rely heavily on an active, moving observer. However, although interface theory is adamant that direct perception is not the case - as I highlight below - because they essentially dodge the problem of phenomenalism, it seems that the question remains open whether "perception" of these lawful perception-action couplings (viz. "affordances") is in fact directly experienced.

\section{Shall we play a game?}

As someone who has dabbled a bit in the world of artificial life (Parisi \& Schlesinger, 2002; Schlesinger, 2004), I have to confess that the high point of interface theory for me is Hoffman et al.'s use of game theory and genetic algorithm (GA) models to bolster the argument. Sadly, GAs in particular are a grossly underused and underappreciated tool in the discipline of psychology, not to mention vision science. In any case, Hoffman et al. have skillfully proposed and analyzed a few well-chosen models, leaving the (open-minded) reader with the strong impression that, just as they suggest, neo-Darwinian mechanisms of change will tilt a perceptual channel or modality not toward perceiving "what's out there," but rather toward "what I need to see to survive." Still, I also see some room for improvement here. For example, it would be very cool to move beyond a relatively "disembodied" model of the observer to one that has at least a very basic sensorimotor system (e.g., an eyeball with a 1-D or 2-D retina, situated in a simple environment and able to change its visual input by rotating). I suspect that even such a modest step toward ecological realism (sorry, pun not intended!) would pay handsome dividends.

\section{An evolutionary promissory note?}

One of the places in the interface theory of perception that I found begging for more detail is the question of precisely how evolution - or more specifically, variation and selectionshapes perceptual experience. In fact, I hoped for and anticipated at least five or six really juicy, compelling, richly articulated just-so stories, each one beginning like this: Imagine the ancestors of the antelope. This creature lived in a hot, dry savannah, much like the Africa of today. Most of its day was spent searching for water, while avoiding large, fierce, predatory beasts. . . . The goal, of course, of these colorful scenarios would be to provide an intuition pump that would map our own everyday experiences onto the world of these imaginary creatures and, ultimately, give us a handle on what it would mean that our perception is determined by fitness rather than by objective reality. Instead, no such anecdotes are offered, but instead, all of the interesting and important work is offloaded to a "Darwin machine" that magically and quietly churns away in the background. For sure, I hope that Hoffman et al. return to this fertile landscape and fill in what looks to me like a rather large hole in their story.

\section{Can you say "qualia"?}

A second unfulfilled expectation is that, despite all the time and effort spent focusing on the nature of perceptual experience, the question of where and how perceptual qualities originate (i.e., phenomenalism) is largely avoided by the interface theory. This is perhaps also a missed opportunity. Specifically, Hoffman et al. have the option of siding with theorists like O'Regan and Noë (2001), who propose that phenomenal experience is simply an awareness of the particular spatiotemporal contingencies that occur during perception-action couplings. In other words, given that interface theory already presupposes that perception and action are dynamically linked, it is only one small step forward to suggest that perceiving these lawful (or contingent or statistically predictable) regularities is ipso facto what constitutes the qualities of perceptual experience, such as "roundness" or "redness."

\section{Darwin and beyond}

I conclude here by noting one last facet of the interface theory that I hope will be elaborated in greater detail someday: In addition to highlighting the problem of offloading much of the heavy lifting to a background Darwin machine (i.e., the mechanisms of change), I also note that the machine itself seems quite outdated. In other words, it is a bit passé merely to wave one's hands glibly and assert, "this skill is important for survival, so obviously natural selection would favor it," or perhaps the more subtle claim, "an organism without this ability would be unlikely to survive." Indeed - and keep in mind that I am not an evolutionary biologist — over the last 50 years, the basic framework of neo-Darwinian evolution has experienced a revolution of its own. A key idea is that a particular phenotype is not selected in isolation, but rather as part of a complex system of structures and behaviors, such that changing one part of the organism has far-reaching and cascading effects throughout the body and genome as a whole. In addition, the idea of organisms as systems is related to the concept of genetic regulatory networks and phenomena such as epigenesis. My point here is not to suggest that the interface theory needs to incorporate such relatively esoteric concepts, but rather, that causally linear claims like "such-and-such will increase fitness" need to be revised and updated in light of the nonlinear reality where such changes actually occur. 


\section{References}

Hoffman, D. D., Singh, M., \& Prakash, C. (2014). The interface theory of perception. Psychonomic Bulletin \& Review.

O'Regan, J. K., \& Noë, A. (2001). A sensorimotor account of vision and visual consciousness. Behavioral and Brain
Sciences, 24, 939-973. doi:10.1017/S0140525X01000115. disc. $973-1031$.

Parisi, D., \& Schlesinger, M. (2002). Artificial life and Piaget. Cognitive Development, 17, 1301-1321.

Schlesinger, M. (2004). Evolving agents as a metaphor for the developing child. Developmental Science, 7, 158-164. 